\title{
Characterization of DNA-Nanopore Interactions by Molecular Dynamics
}

\author{
Abhijit Ramachandran ${ }^{1}$, Yaling Liu ${ }^{1,2 *}$, Waseem Asghar ${ }^{3,4}$, and Samir M. Iqbal ${ }^{1,3,4}$ \\ ${ }^{1}$ Biomedical Engineering, \\ ${ }^{2}$ Mechanical and Aerospace Engineering, \\ ${ }^{3}$ Nanotechnology Research and Teaching Facility, \\ ${ }^{4}$ Department of Electrical Engineering, University of Texas at Arlington, Arlington, TX, 76019 \\ *Corresponding Author: \\ Yaling Liu \\ Department of Mechanical and Aerospace Engineering \\ University of Texas at Arlington \\ Arlington, TX, 76019 \\ Phone: $817-272-1256$ \\ Email: yaling.liu@uta.edu
}

Received: 25 March 2009; | Revised: 29 May 2009; | Accepted: 25 June 2009

\begin{abstract}
A major challenge in using solid-state nanopores for DNA detection and sequencing is the molecular selectivity and sensitivity resolution through proper control of DNA-nanopore interface. This paper explores the interaction mechanisms between DNA and nanopores through molecular dynamics simulations. The DNA translocation dynamics through nanopores of various diameters and under various applied biasvoltages are characterized. Such property-function relationship can be used for the optimal designs of the lab-on-chip DNA diagnostic devices.
\end{abstract}

Keywords: Nanopore; DNA; Molecular Dynamics; Molecular Interaction.

\section{Introduction}

Biological nanopores like alpha-hemolysin channels have been used extensively to study DNA translocation ${ }^{1-4}$. A number of important studies have looked at statistically discriminating between different sequences of oligonucleotides, or identifying parameters that govern the analyte translocation through these pores (e.g. biasing voltage, salinity, $\mathrm{pH})^{5,6}$. The major disadvantages of biological protein channels are fixed size and limited stability, which stimulated the development of solid-state nanopores ${ }^{7}$. Advances in Micro/Nanofabrication technology has now made it possible to make solid-state nanopores with precise control on size (diameter, length of pores/channels) and surface material properties ${ }^{8-10}$.

The understanding of the dynamics of DNA in nanopores is important for the development of lab-on-chip devices for biomolecular analysis. 
However, the interaction between DNA and nanopores is still not well understood due to the small length scales of the DNA/nanopore and the dynamic nature of the translocation process. Studies of electrophoretic transport of DNA in nanochannels have revealed that DNA-channel surface interaction leads to a diffusion rate much lower than predictions by traditional diffusion theory ${ }^{11}$. Extensive experiments and simulations have focused on understanding translocation of a long DNA strand by analyzing the dips in the ionic current due to the blockage of the channel and correlating the time of dip in the ionic current to the polymer length ${ }^{9,12-17}$. There has also been work done to understand the stretching of the DNA in the nanopore under the application of electric field ${ }^{3,18}$, as well as the effect of temperature and DNA sequence on the translocation kinetics ${ }^{19}$. All these works have a common feature in that they have considered a DNA with length greater than that of the nanopore and initially located outside the pore. It is known that the translocation kinetics outside and inside the pore are different due to the ion accumulation near the pore entrances. Moreover in the reported simulation studies, higher voltages (compared to typical experiments) are applied to overcome the timescale limitations. In this study, periodic boundary conditions are applied at the inlet and outlet of the pore. Thus, there is no ion depletion under higher voltages and DNA is always within the pore. This helps characterize translocation kinetics of DNA inside a nanopore and DNAnanopore interaction without the entrance and exit effect.

Nanopores with molecular selectivity are needed to determine DNA conformation and base pair level information simultaneously ${ }^{20}$. To provide such selectivity, simple chemical treatment such as silane coating will not be sufficient $^{21-24}$. Iqbal et al. ${ }^{10}$ have demonstrated that such selectivity can be imparted by using tethered DNA hair-pin loops coated on the inner surface of the nanopore. But little is known about properties of these coated DNA monolayers which are influenced by size, geometry, and surface curvature of the pore ${ }^{25}$. Other factors that also modulate the tethered DNA orientation include initial orientation, attachments with the wall, applied electric field, and the hydrodynamic interactions ${ }^{19,}{ }^{26}$. Thus, it is important to characterize the interaction between DNA and chemically modified nanopores.

The goal of this paper is to develop an understanding of the interaction between DNA and nanopore surfaces and the translocation process of DNA by probing the DNA-nanopore interaction mechanisms through molecular dynamics simulations. This research will not only advance the molecular-level understanding of the DNA-nanopore interface, but also help design labon-chip devices for diagnosis and measurement of therapeutic responses.

\section{Method}

Molecular Dynamics (MD) simulations are performed on NAMD ${ }^{27}$ (NAnoscale Molecular Dynamics) to study DNA translocation in nanopores of various diameters and surface coatings. The CHARMM ${ }^{28}$ force field is used for the DNA, nanopore (silicon nitride), and their interactions. The total potential energy is composed of bonded energies and non-bonded pair interaction energies:

$$
\begin{aligned}
& U=E_{\mathrm{bond}}+E_{\mathrm{angle}}+E_{\mathrm{dihedral}}+E_{\mathrm{improper}}+E_{\mathrm{elec}}+E_{\mathrm{vdw}}+E_{\text {constrain }}+E_{\mathrm{other}} \\
& =\sum k_{b}\left(b-b_{0}\right)^{2}+\sum k_{\theta}\left(\theta-\theta_{0}\right)^{2}+\sum k_{\chi}(1+\cos (n(\chi-\delta))) \\
& +\sum k_{\psi}\left(\psi-\psi \psi_{0}\right)^{2}+\sum_{i<j} \frac{q_{i} q_{j}}{4 \pi \varepsilon_{0} r_{i j}}+\sum_{i<j}\left(\frac{A_{i j}}{r_{i j}{ }^{12}}-\frac{B_{i j}}{r_{i j}{ }^{6}}\right)+E_{\text {constrain }}+E_{\text {other }}
\end{aligned}
$$

Non-bonded interactions are cut off at $1.2 \mathrm{~nm}$ and the Lennard-Jones potential is smoothly shifted to zero between $1 \mathrm{~nm}$ and the cutoff distance. The pair list is updated every step using a $1.4 \mathrm{~nm}$ cutoff. Particle mesh Ewald (PME) is applied for long range electrostatics. The temperature is set at 
be $295 \mathrm{~K}$ and a time step of $1 \mathrm{fs}$ is used. Periodic boundary conditions are applied at the entrance and outlet of the pore. Analysis and visualizations are done with Visual Molecular Dynamics $(\mathrm{VMD})^{29}$. All simulations are performed on the supercomputer cluster POPEL at Pittsburgh Supercomputing Center $(\mathrm{PSC})^{30}$. A 2 ns simulation on 8 processors took around 24 hours. The number of atoms in the system varies with the size of the nanopore. A typical simulated system has 14814 atoms, consisting of 8000 for nanopore, 6383 for water, 258 for DNA and 200 for ions. The timescale covered by molecular dynamics simulations is currently limited to $100 \mathrm{~ns}^{31}$. To accelerate the translocation events that normally take milliseconds, the MD simulations are performed at a higher applied voltage than 100 $200 \mathrm{mV}$, applied typically in experiments.
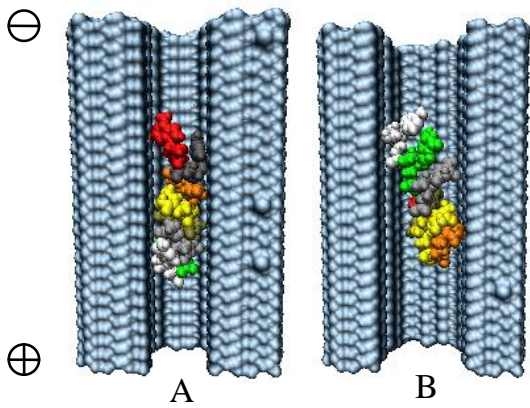

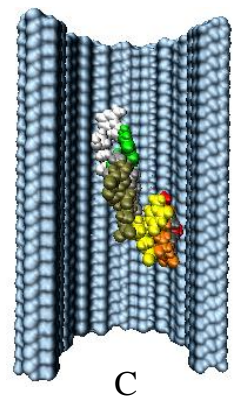

$\mathrm{C}$

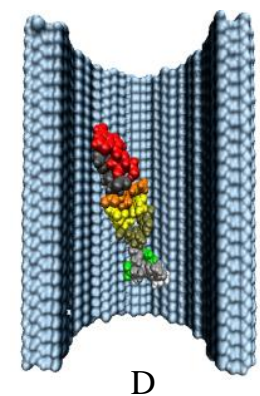

D

Figure 1. DNA translocation in pores of sizes: (A) $1.5 \mathrm{~nm}$; (B) $2 \mathrm{~nm}$; (C) $3 \mathrm{~nm}$; (D) $4 \mathrm{~nm}$.

\section{Result}

\subsection{Size-Dependent DNA-Nanopore interaction}

In the absence of surface friction, nanochannels would be a free solution environment in which DNA molecules would move with a length-independent mobility. However, electrophoretic mobility of DNA molecules in slit-like nanochannels has been observed to be length dependent ${ }^{11}$. This clearly indicates that molecular interactions with the confining walls are significant, and the notion of free solution electrophoresis breaks down. To understand the size-dependent translocation, DNA interaction with pores of various sizes is studied here. A short single strand DNA (ss-DNA) of 8 bases (AATTGTGA) is driven by electrophoresis through nanopores with diameters ranging from $1.5 \mathrm{~nm}$ to $4 \mathrm{~nm}$, as shown in Fig. 1 (A: Adenosine, T: Thymine, G: Guanine, C: Cytosine). The water molecules and ions are not shown in the figure for clarity.

The van der Waals (vdW) force between the DNA and nanopore, the translocation velocity of DNA, and the ionic current as functions of pore size are plotted in Fig. 2 (A), (B), (C), respectively. From Fig. 2(A), DNA-nanopore interaction force decreases as the pore diameter increases, due to the reduced confinement effect. Accordingly, DNA translocation velocity increases as the pore size increases. However, both the translocation velocity and van der Waals force do not change linearly with size change, but satisfy an exponential curve. There is a gradual increase in translocation velocity as nanopore diameter increases at the beginning. It then reaches a constant limit indicating that an increase in the pore diameter beyond $3 \mathrm{~nm}$ will not affect the ssDNA translocation process anymore. Establishing such nanopore size - DNA translocation velocity relationship can help design nanopore size for optimized signal yield. The ionic current increases with the increase of pore diameter, satisfying a parabolic curve. It should be noted that periodic boundary conditions are applied at the inlet and outlet of the pore, thus there is no depletion of ions as is usually observed in nanopore experiments when higher voltage is applied. Although experimental data suggests that the translocation time is different for long DNA 
strands with different sequences ${ }^{31,32}$, we did not observe significant difference in translocation dynamics for homopolymers poly-dA or poly-dT through the nanopores and believe that it might be due to the small strand ( 8 base) ssDNA used in the simulation. Furthermore, it is expected that there is an optimal pore diameter for a particular voltage bias applied, which would provide sufficient confinement to enhance the molecule detection yet large enough for DNA to pass through. Such optimal designs will be explored in the future work.
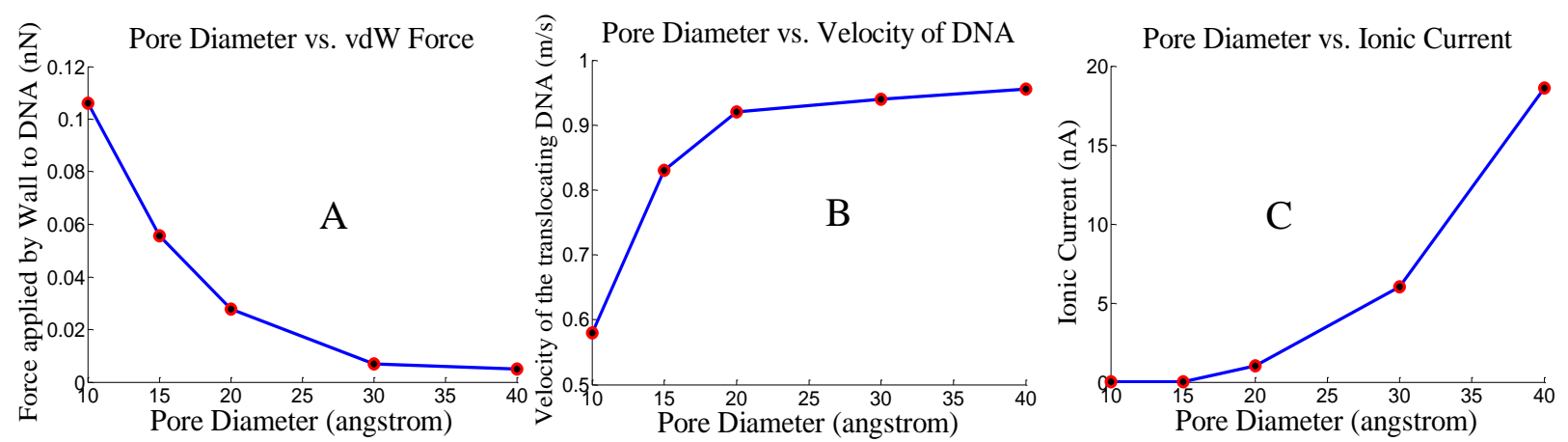

Figure 2. DNA translocation in pores of different sizes (A) van der Waals force; (B) Velocity;

(C) Ionic current.
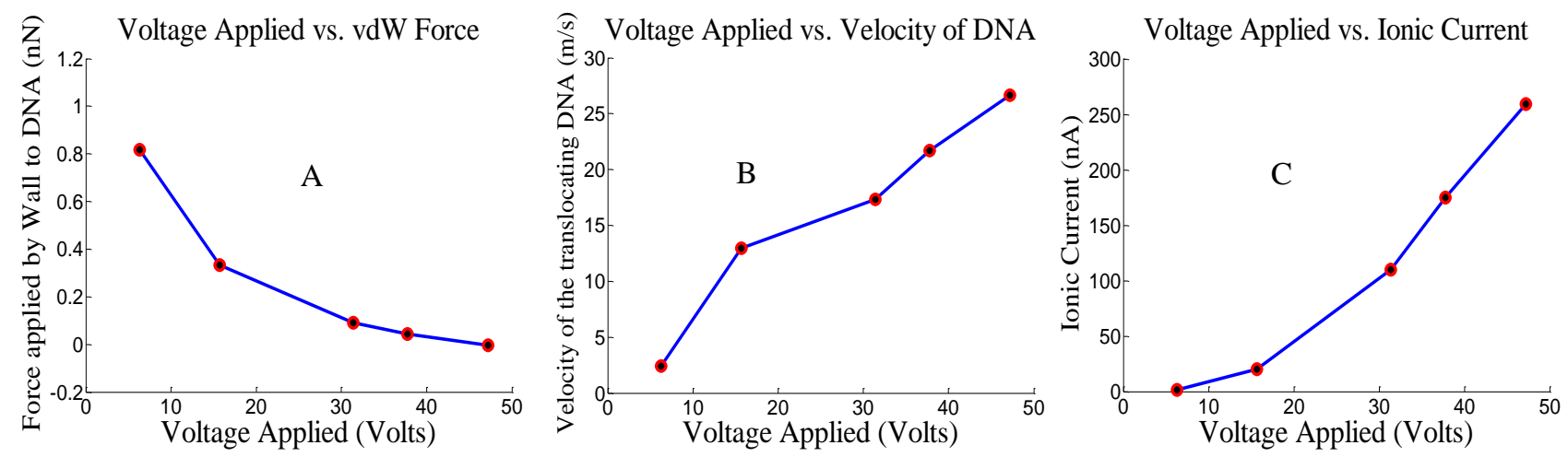

Figure 3. DNA translocation under different applied voltages (A) van der Waals force; (B) Velocity;

(C) Ionic current.

\subsection{Voltage-Dependent DNA-Nanopore Interaction}

Besides nanopore diameter, the applied voltage also largely influences the DNA translocation speed. Considering a balance between confinement and translocation, a nanopore with a diameter of $2 \mathrm{~nm}$ is chosen and is applied with various voltages. From Fig. 3, a nearly linear trend can be observed between applied voltage and translocation velocity, while a parabolic trend is observed between applied voltage and vdW force or ionic current. Ideally, ionic current should increase linearly with the increase of applied voltage for free ions passing through a nanopore. The nonlinear relationship observed in Fig. 3(C) might be induced by the existence of DNA. The free current increases with applied voltage while DNA velocity also increases. Faster moving DNA is carrying more charges due to ion condensation as well as electrokinetic flow. It should be noted that a high voltage 
is applied to observe the translocation in less than 100 ns. To study the translocation at lower voltages a coarse grained DNA model is needed, which will be included in future work.

\subsection{Nanopores with Surface Functionalizations}

Coating nanopores with DNA or other organic molecules like silanes can make nanopores more biologically friendly and provide control over surface charges, hydrophobicity, and chemical functionality. It is important to characterize the surface property, size, and orientation of the modified surface. The coatings used in our case are 8-base long single strands of DNA. The tethered DNA carry a negative backbone, thus these re-orient and are stretched under the applied bias voltage. Characterization of the coating polymer re-orientation is essential to predict the effective pore diameter under a particular applied voltage, which influences DNA translocation process. The spacing between the attached ss-DNA molecules on nanopore surface is determined by their radius of gyration. To avoid tethered DNA from peeling off, nucleotide of DNA strand closest to the wall are fixed on the nanopore surface. The charge on each tethered DNA is assumed " $-e$ ", due to the phosphate backbone.
Under the applied voltage bias, translocation of ions towards the opposite electrodes is observed. Since the coated ss-DNA carries a -e charge, these curled tethered ss-DNA begin to get straightened in the direction of the oppositely charged electrode. The straightened DNA increases the effective pore diameter, which is measured as the empty space in the nanopore not occupied by the coated ss-DNA. After the system reaches equilibrium, the distances between opposing ss-DNA molecules are calculated and averaged to get the effective pore diameter. There are many factors that contribute to the effective pore diameter, such as the flow of ions, the ion condensation around the DNA, voltage bias applied, etc. The effective pore diameters are plotted as a function of the applied voltage bias in Fig. 4. The effective pore diameter increases with the increase of applied electric field strength. The kinetics of DNA translocation in a polymer coated pore differs from that in a bare nanopore. In a bare nanopore, ions inside the nanopore form a double layer which prevents DNA translocation at a faster speed within the pore. With a tethered DNA coating on the surface, the ion layers disappear and different translocation speeds are expected.
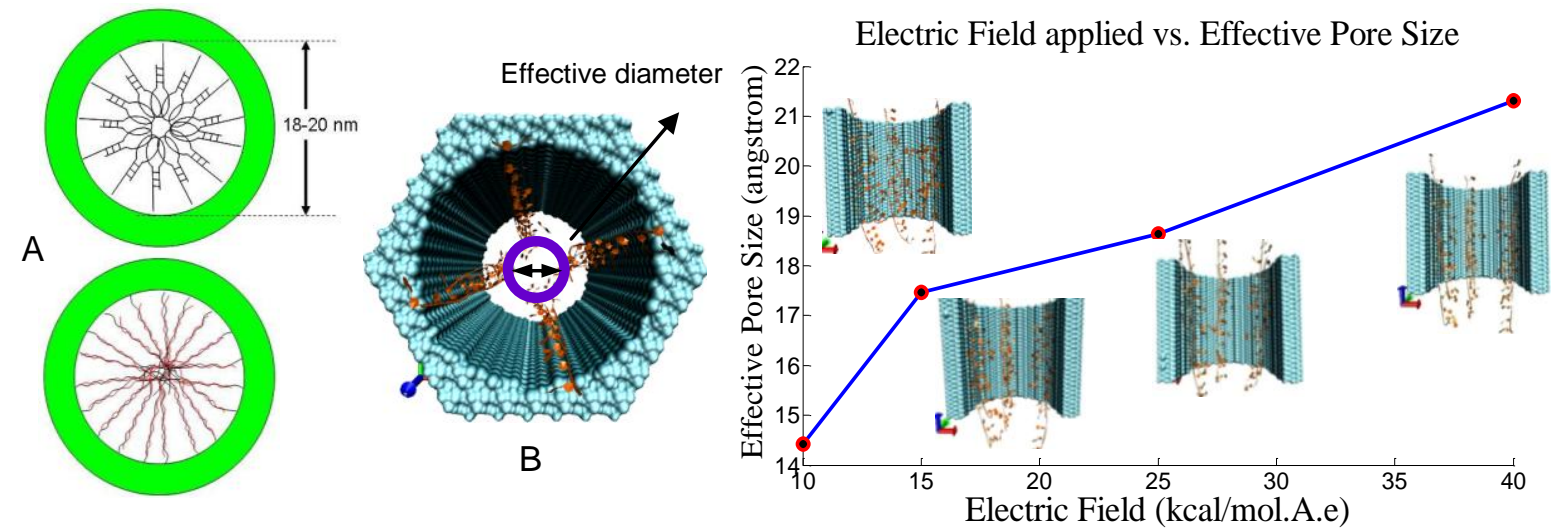

Figure 4. ss-DNAs coated on nanopore surface. (A) Radii of gyration for same length ss-DNA in hairpin and linear conformation, coated inside a pore; (B) Effective pore diameter; (C) DNA reorient under applied Electric Field of various strengths and effective pore diameter as a function of Electric Field strength.

\subsection{Need for Coarse-grained Model}

MD simulations are capable of capturing essentially all of the phenomena in the DNAnanochannel interaction, but are limited to a few thousands atoms - representing only a tiny portion of a long DNA chain $\left(10^{2}\right.$ bp with water, ions, and pore molecules considered). In addition, a very small time step on the order of femtosecond is 
required to accommodate the inherent high vibrational frequencies of light atoms. As a result, MD simulations are also limited to nanosecond time scale. To study a long DNA translocation in nanopore such as 6000 bp DNA under an applied voltage of around 100-200 mV (typically used in experiments), a coarse grained DNA model is needed. Such coarse-grained model for DNA can be established by first grouping atoms into fewer interaction sites, followed by the construction of an equivalent inter-site interaction potential. For example, a Thymine nucleotide, comprising of 32 atoms, can be coarse-grained into 3 interaction sites, one each for the phosphate $(\mathrm{P})$, sugar $(\mathrm{S})$, and base. There are four types of base sites, one for each type of base in DNA. To construct an equivalent inter-site interaction potential, a standard procedure ${ }^{33}$ is to parameterize a preselected analytical function by matching the thermodynamic properties drawn from the fullatom MD simulations and experiments.

\section{Conclusion}

The translocation speed of DNA in a nanopore depends on physical parameters such as nanopore diameter, electrophoretic bias, and surface coatings. The orientation of the tethered DNA in the nanopore changes the effective nanopore diameter. The effective nanopore diameter is found to be controllable in two ways: the bare nanopore size during fabrication and the strength of the applied electric field. This modelbased system can be used to optimize parameters in the design of nanopore systems for DNA/gene sequencing. The major drawback of full atomistic molecular simulation is the limitation of timescale with $100 \mathrm{~ns}$. In the future work, a coarse grained DNA model will be used to simulate low-voltage DNA translation with timescale in milliseconds.

\section{Acknowledgment}

Y. Liu acknowledges the support of this work from University of Texas at Arlington Research Enhancement Program and the National Science Foundation TeraGrid supercomputing cluster. S.M. Iqbal acknowledges support from the Dallas
Metroplex Research Consortium for Electronic Devices and Materials (MRCEDM).

\section{References}

1. Gu, L. Q.; Cheley, S.; Bayley, H., Capture of a single molecule in a nanocavity. Science 2001, 291, (5504), 636-640.

2. Mathe, J.; Aksimentiev, A.; Nelson, D. R.; Schulten, K.; Meller, A., Orientation discrimination of single-stranded DNA inside the alpha-hemolysin membrane channel. Proceedings of the National Academy of Sciences of the United States of America 2005, 102, (35), 12377-12382.

3. Muthukumar, M.; Kong, C. Y., Simulation of polymer translocation through protein channels. Proceedings of the National Academy of Sciences of the United States of America 2006, 103, (14), 5273-5278.

4. Kasianowicz, J. J.; Brandin, E.; Branton, D.; Deamer, D. W., Characterization of individual polynucleotide molecules using a membrane channel. Proceedings of the National Academy of Sciences of the United States of America 1996, 93, (24), 13770-13773.

5. DeGuzman, V. S.; Lee, C. C.; Deamer, D. W.; Vercoutere, W. A., Sequence-dependent gating of an ion channel by DNA hairpin molecules. Nucleic Acids Research 2006, 34, (22), 64256437.

6. Bockelmann, U.; Viasnoff, V., Theoretical study of sequence-dependent nanopore unzipping of DNA. Biophysical Journal 2008, 94, (7), 2716-2724.

7. Dekker, C., Solid-state nanopores. Nature Nanotechnology 2007, 2, (4), 209-215.

8. Li, J. L.; Gershow, M.; Stein, D.; Brandin, E.; Golovchenko, J. A., DNA molecules and configurations in a solid-state nanopore microscope. Nature Materials 2003, 2, (9), 611-615.

9. Zhao, X. C.; Payne, C. M.; Cummings, P. T.; Lee, J. W., Single-strand DNA molecule translocation through nanoelectrode gaps. Nanotechnology 2007, 18, (42), 424018424024. 
10. Iqbal, S. M.; Akin, D.; Bashir, R., Solid-state nanopore channels with DNA selectivity. Nature Nanotechnology 2007, 2, (4), 243-248.

11. Cross, J. D.; Strychalski, E. A.; Craighead, H. G., Size-dependent DNA mobility in nanochannels. Journal of Applied Physics 2007, 102, (2), 024701-024705

12. Wanunu, M.; Sutin, J.; McNally, B.; Chow, A.; Meller, A., DNA Translocation Governed by Interactions with Solid-State Nanopores. Biophysical Journal 2008, 95, (10), 47164725.

13. Meller, A., Dynamics of polynucleotide transport through nanometre-scale pores. Journal of Physics-Condensed Matter 2003, 15, (17), R581-R607.

14. Chen, P.; Gu, J. J.; Brandin, E.; Kim, Y. R.; Wang, Q.; Branton, D., Probing single DNA molecule transport using fabricated nanopores. Nano Letters 2004, 4, (11), 2293-2298.

15. Heng, J. B.; Aksimentiev, A.; Ho, C.; Marks, P.; Grinkova, Y. V.; Sligar, S.; Schulten, K.; Timp, G., The electromechanics of DNA in a synthetic nanopore. Biophysical Journal 2006, 90, (3), 1098-1106.

16. Meller, A.; Nivon, L.; Branton, D., Voltagedriven DNA translocations through a nanopore. Physical Review Letters 2001, 86, (15), 3435-3438.

17. Aksimentiev, A.; Heng, J. B.; Cruz-Chu, E. R.; Timp, G.; Schulten, K., Microscopic kinetics of DNA translocation through synthetic nanopores. Biophysical Journal 2005, 88, (1), 352a-352a.

18. Heng, J. B.; Aksimentiev, A.; Ho, C.; Marks, P.; Grinkova, Y. V.; Sligar, S.; Schulten, K.; Timp, G., Stretching DNA using the electric field in a synthetic nanopore. Nano Letters 2005, 5, (10), 1883-1888.

19. Fyta, M. G.; Melchionna, S.; Kaxiras, E.; Succi, S., Multiscale coupling of molecular dynamics and hydrodynamics: Application to DNA translocation through a nanopore. Multiscale Modeling \& Simulation 2006, 5, (4), 1156-1173.

20. Fologea, D.; Brandin, E.; Uplinger, J.; Branton, D.; Li, J., DNA conformation and base number simultaneously determined in a nanopore. Electrophoresis 2007, 28, (18), 3186-3192.

21. Kasianowicz, J. J.; Nguyen, T. L.; Stanford, V. M., Enhancing molecular flux through nanopores by means of attractive interactions. Proceedings of the National Academy of Sciences of the United States of America 2006, 103, (31), 11431-11432.

22. Hogg, T.; Zhang, M.; Yang, R., Modeling and Analysis of DNA Hybridization Dynamics at Microarray Surface in Moving Fluid. IEEE International Conference on Robotics and Automation 2008, 3419-3424.

23. Chan, V.; Graves, D. J.; McKenzie, S. E., The biophysics of DNA hybridization with immobilized oligonucleotide probes. Biophysical Journal 1995, 69, (6), 2243-2255.

24. Smeets, R. M. M.; Keyser, U. F.; Krapf, D.; Wu, M. Y.; Dekker, N. H.; Dekker, C., Salt dependence of ion transport and DNA translocation through solid-state nanopores. Nano Letters 2006, 6, (1), 89-95.

25. Hofler, L.; Gyurcsanyi, R. E., Coarse grained molecular dynamics simulation of electromechanically-gated DNA modified conical nanopores. Electroanalysis 2008, 20, (3), 301-307.

26. Wang, G. M.; Sandberg, A. C., Nonequilibrium all-atom molecular dynamics simulations of free and tethered DNA molecules in nanochannel shear flows. Nanotechnology 2007, 18, (13), 135702135710.

27. Kale, L.; Skeel, R.; Bhandarkar, M.; Brunner, R.; Gursoy, A.; Krawetz, N.; Phillips, J.; Shinozaki, A.; Varadarajan, K.; Schulten, K., NAMD2: Greater scalability for parallel molecular dynamics. Journal of Computational Physics 1999, 151, (1), 283312.

28. MacKerell, A. D.; Bashford, D.; Bellott, M.; Dunbrack, R. L.; Evanseck, J. D.; Field, M. J.; Fischer, S.; Gao, J.; Guo, H.; Ha, S.; JosephMcCarthy, D.; Kuchnir, L.; Kuczera, K.; Lau, F. T. K.; Mattos, C.; Michnick, S.; Ngo, T.; Nguyen, D. T.; Prodhom, B.; Reiher, W. E.; Roux, B.; Schlenkrich, M.; Smith, J. C.; Stote, R.; Straub, J.; Watanabe, M.; WiorkiewiczKuczera, J.; Yin, D.; Karplus, M., All-atom 
empirical potential for molecular modeling and dynamics studies of proteins. Journal of Physical Chemistry B 1998, 102, (18), 35863616.

29. Humphrey, W.; Dalke, A.; Schulten, K., VMD: Visual molecular dynamics. Journal of Molecular Graphics 1996, 14, (1), 33-38.

30. Catlett, C.; Allcock, W. E.; Andrews, P.; Aydt, R.; Bair, R.; Balac, N.; Banister, B.; Barker, T.; Bartelt, M.; Beckman, P. e. a., TeraGrid: Analysis of Organization, System Architecture, and Middleware Enabling New Types of Applications. HPC and Grids in Action. High Performance Computing and Grids in Action 2008, 16, Edited by: Grandinetti L. Amsterdam: IOS Press.
31. Aksimentiev, A.; Heng, J. B.; Timp, G.; Schulten, K., Microscopic kinetics of DNA translocation through synthetic nanopores. Biophysical Journal 2004, 87, (3), 2086-2097.

32. Meller, A.; Nivon, L.; Brandin, E.; Golovchenko, J.; Branton, D., Rapid nanopore discrimination between single polynucleotide molecules. Proceedings of the National Academy of Sciences of the United States of America 2000, 97, (3), 1079-1084.

33. Knotts, T. A.; Rathore, N.; Schwartz, D. C.; de Pablo, J. J., A coarse grain model for DNA. Journal of Chemical Physics 2007, 126, (8), 084901-0849012. 